\title{
Cancer cells compress intratumour vessels
}

\author{
Pressure from proliferating cells impedes transport of therapeutic drugs into tumours.
}

T he delivery of therapeutic drugs to solid tumours may be impaired by structural and functional abnormalities in blood and lymphatic vessels ${ }^{1}$. Here we provide evidence that proliferating cancer cells cause intratumour vessels to compress and collapse. By reducing this compressive mechanical force and opening vessels, cytotoxic cancer treatments have the potential to increase blood perfusion, thereby improving drug delivery.

Elevated interstitial fluid pressure - a hallmark of solid tumours - is commonly assumed to compress intratumour vessels. However, the interstitial fluid pressure is about equal to the microvascular pressure in tumours, making it unlikely that the collapse of permeable vessels is mediated by fluid pressure $^{2}$. We therefore investigated whether proliferating cancer cells might cause compression of tumour vessels and, if so, whether relieving this compression would allow them to open and regain their functionality.

To relieve compression, we targeted tumour cells with diphtheria toxin, which is much less cytotoxic to mouse than to human cells $^{3}$ (see supplementary information for methods). Diphtheria toxin had no effect on murine tumours (T-241 type), normal murine tissue (liver) or endothelium, but caused apoptosis and time-dependent regression in human-tumour xenografts (HSTS-26T type) grown in mice (results not shown).

We found a greater fraction of blood and lymphatic vessels with an open lumen in diphtheria-toxin-treated human tumours than in controls treated with PBS buffer (Fig. 1a-c). Treatment with diphtheria toxin also causes vessels to have a rounder crosssection in the human tumours but has no effect on vessel morphology in the murine tumours. Compared with collapsed vessels, open vessels are surrounded by fewer cells, suggesting that the local compressive force is lower (Fig. 1d). The diameter of perfused blood vessels increased in the human tumours after treatment with diphtheria toxin and, in contrast to results obtained after treatment with taxane ${ }^{4}$, a greater fraction of blood vessels were perfused.

These changes in vascular morphology cannot be explained by a difference in collagen I content or in the molecular determinants of blood- and lymphatic-vessel formation and remodelling (namely, vascular endothelial growth factor, vascular endothelial growth factor-C, vascular endothelial growth factor receptor-2, angiopoietin-1 or angiopoietin-2). Vessels associated with smooth-muscle $\alpha$-actin-expressing cells or with a prominent collagen IV basement membrane are more likely to have an open lumen. Lymphatic vessels are rarely adjacent to smooth-muscle $\alpha$-actin-expressing cells.
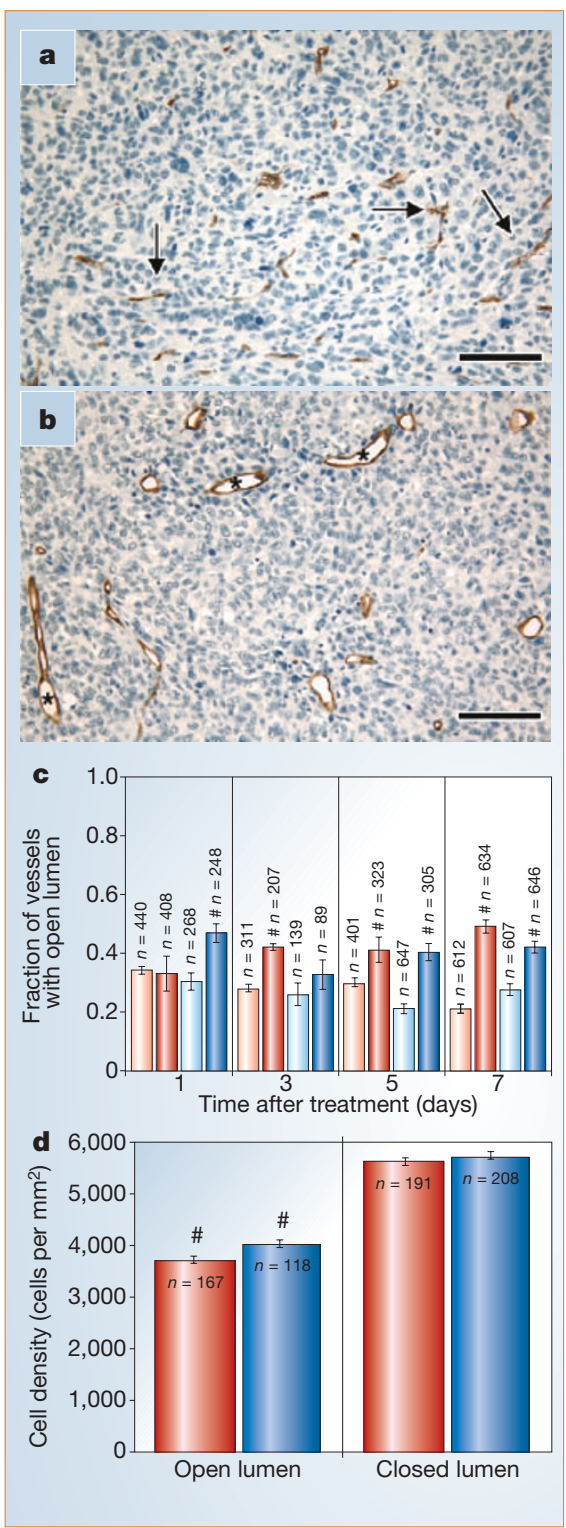

Figure 1 Collapsed blood and lymphatic vessels open after relieving compressive forces generated by cancer cells in human tumours. a, Blood vessels in HSTS-26T tumours remain collapsed (arrows) 5 days after treatment with PBS buffer. b, A greater fraction of blood vessels have an open lumen (asterisks) 5 days after treatment with diphtheria toxin. Scale bars, $50 \mu \mathrm{m}$. Results were similar for lymphatic vessels. c, Fraction of blood vessels (red bars) and lymphatic vessels (blue bars) with an open lumen at different times after treatment with diphtheria toxin (dark bars) or PBS buffer (light bars). d, Blood vessels (red bars) and lymphatic vessels (blue bars) with an open lumen have a lower surrounding cell density compared with vessels that have a collapsed lumen. Hash symbols indicate that $P<0.05$.
We next tested whether intratumour lymphatic vessels, which are ordinarily nonfunctional or absent ${ }^{5,6}$, become functional upon opening. We found, surprisingly, that those inside diphtheria-toxin-treated tumours did not become functional, as judged by fluorescence or ferritin microlymphangiography ${ }^{6}$. It is possible that tumours permanently damage lymphaticvessel structures, such as lymphatic endothelial microvalves, or that the lack of pulsatile blood flow in tumours inhibits lymph formation ${ }^{7}$.

The tumour margin, in which mechanical stress is predicted to be lower (see supplementary information), contained functional lymphatic vessels, as well as a greater fraction of open lymphatics than was found in intratumour regions (data not shown). Regions subject to lower predicted compressive forces thus contain open, functional lymphatics, whereas regions exposed to greater compressive forces contain collapsed, nonfunctional lymphatic vessels.

The role of a tumour's mechanical microenvironment in its progression and treatment is starting to emerge. For example, compressive forces inhibit tumour cell growth $^{8}$ and upregulate adhesion molecules ${ }^{9}$. Clinically, tumours can compress large vessels and the spinal cord.

Our findings indicate that proliferating tumour cells can also cause intratumour vessels to collapse, particularly those without supportive stromal structures, leading to impaired function. Tumour-specific cytotoxic therapy may result in more efficient drug (or nutrient) delivery by decompressing collapsed vessels ${ }^{10}$. Whether these newly perfused vessels also provide additional routes for metastasis is not yet known.

Timothy P. Padera, Brian R. Stoll, Jessica B. Tooredman, Diane Capen, Emmanuelle di Tomaso, Rakesh K. Jain Edwin L. Steele Laboratory, Department of Radiation Oncology, Massachusetts General Hospital, Harvard Medical School, Boston,

Massachusetts 02114, USA

e-mail: jain@steele.mgh.harvard.edu

1. Jain, R. K. Nature Med. 9, 685-693 (2003).

2. Boucher, Y. \& Jain, R. K. Cancer Res. 52, 5110-5114 (1992)

3. Arbiser, J. L. et al. Am. J. Pathol. 155, 723-729 (1999).

4. Griffon-Etienne, G., Boucher, Y., Brekken, C., Suit, H. D. \& Jain, R. K. Cancer Res. 59, 3776-3782 (1999).

5. Alitalo, K. \& Carmeliet, P. Cancer Cell 1, 219-227 (2002).

6. Padera, T. P. et al. Science 296, 1883-1886 (2002).

7. Schmid-Schönbein, G. W. Lymph. Res. Biol. 1, 25-31 (2003).

8. Helmlinger, G., Netti, P. A., Lichtenbeld, H. C., Melder, R. J. \&

Jain, R. K. Nature Biotechnol. 15, 778-783 (1997).

9. Koike, C. et al. Br. J. Cancer 86, 947-953 (2002).

10. Reinhold, H. S. Eur. J. Cancer 7, 273-280 (1971).

Supplementary information accompanies this communication on Nature's website.

Competing financial interests: declared none. 